\title{
EI APRENDIZAJE SERVICIO Y EL DESARROLLO DE LAS COMPETENCIAS EMOCIONALES EN LA FORMACIÓN INICIAL DEL PROFESORADO
}

\author{
Mayka García García \\ Laura Sánchez Calleja \\ Universidad de Cádiz
}

\begin{abstract}
RESUMEN: El presente trabajo ${ }^{1}$ tiene como objetivo principal hacer visible y analizar la conciencia del desarrollo emocional del alumnado del Grado de Educación Infantil de la Universidad de Cádiz, que participa en las experiencias de Aprendizaje y Servicio que se realizan dentro de un itinerario que persigue la institucionalización curricular ${ }^{2}$ del mismo. Para ello empleamos una metodología de corte cualitativa, donde los relatos personales de los/as estudiantes -como instrumentos de recogida de información- son analizados a través de un sistema categorial previo. Los resultados muestran, ilustrados a través de la propia voz del alumnado, el desarrollo de sus competencias emocionales, lo cual nos permite apuntar que el Aprendizaje y Servicio permite andamiar esta dimensión en el contexto de la formación inicial del profesorado favoreciendo su desarrollo intrapersonal e interpersonal.
\end{abstract}

PALABRAS CLAVE: Aprendizaje y Servicio, educación emocional, formación inicial del profesorado.

\section{LEARNING SERVICE AND DEVELOPMENT OF EMOTIONAL COMPETENCIES IN INITIAL TEACHER TRAINING}

\footnotetext{
ABSTRACT: This work has as main objective to make visible and analyze awareness of the emotional development of students Grade Early Childhood Education at the University of Cádiz, which are involved in Service Leaning experiences done within a pathway chasing curriculum institutionalization

1. Se adscribe a un proyecto específico denominado Haciendo visible el desarrollo de las competencias emocionales a través del Aprendizaje Servicio, financiada por la convocatoria Actúa 2015/2016 de la

2. Éste se viene desarrollando desde 2013/2014 y se lleva a cabo en tres asignaturas distintas del ámbito de la atención a la diversidad, en $2^{\circ}, 3^{\circ}$ y $4^{\circ}$ curso del Grado de Educación Infantil de la Universidad
} Universidad de Cádiz. de Cádiz. 
of it. For it, we use a qualitative methodology approach, where the personal accounts of students - as tools for gathering information- were analyzed through a previous category system. The results show, illustrated through the voice of the students, develop their emotional skills, which allows us to conclude that the scaffold ApS allows this dimension in the context of initial teacher favoring their intrapersonal and interpersonal development.

KEYWORDS: Service-Learning, emotional education, initial teacher training.

Recibido: 20/03/2016

Aceptado: 16/07/2016

Correspondencia: Mayka García García, Universidad de Cádiz, Facultad de Ciencias de la Educación, Campus Universitario de Puerto Real, Avda. República Saharaui s/n, 11510, Puerto Real, Cádiz. Email: mayka.garcia@uca.es.

\section{INTRODUCCIÓN}

En los últimos años se está realizando un esfuerzo importante encaminado a la sistematización, a través de la investigación, de las aportaciones que las propuestas de Aprendizaje y Servicio (en adelante ApS) pueden hacer al desarrollo educativo en todos los niveles, desde la educación infantil hasta la universidad (Batlle, 2013). Es este último contexto, el universitario, y en particular el de la formación inicial del profesorado, al que se adscribe este trabajo. Podemos encontrar trabajos de sumo interés como los de Furco (2001), Tedesco, Hernáiz, Tapia y Rial (2008), Folgueiras y Martínez (2009), Martínez (2010), Mangas y Martínez-Odría, (2012), Rubio, Prats y Gómez (2013), entre otros, que nos ayudan a comprender por qué, para qué y cómo el ApS se ha adentrado en la universidad con tanta facilidad. Ello tiene que ver con las propias características pedagógicas de la propuesta y su flexibilidad (Batlle, 2011; Puig y Palos, 2006; Carbonell, 2015), con sus posibilidades en la articulación de un currículo significativo y en la acción, también en la universidad (Campo, 2006, Francisco y Moliner, 2010; García y Cotrina, 2016), y con la multiplicidad a través de fórmulas diversas de desarrollo, que van desde propuestas particulares en asignaturas concretas a despliegues institucionales (Opazo, Aramburuzabala y Cerrillo, 2016).

Uno de los espacios universitarios donde el ApS está alcanzando una mayor expansión es el de la formación inicial del profesorado, donde su papel en el desarrollo de la educación inclusiva (García y Cotrina, 2012), en la articulación de la justicia social (Murillo y Arambuzabala, 2014), en el acompañamiento educativo (García-Pérez y Mendía, 2015) o como propuesta de innovación docente (Aramburuzabala y García, 2012; Martínez-Domínguez, Domínguez, Sáez, y Amundarain, 2015), se revelan importantes.

Cabe poner de manifiesto que el ApS, en este contexto, puede ser entendido como "un desarrollo socioeducativo y solidario de la metodología de proyectos donde, tanto los/as estudiantes como aquellas otras personas implicadas, aprendemos realizando un servicio a la comunidad" (García y Cotrina, 2015, p. 5). Y es que, a través del 
ApS se trata, principalmente, de aprender haciendo un servicio en beneficio de otras personas, lo que nos mantiene activos y nos permite desarrollar nuestra vertiente personal y profesional más social y humana, tanto a estudiantes como al profesorado implicado. En este sentido, podemos intuir que las competencias emocionales (Bisquerra y Pérez Escoda, 2007) emergen de forma directa e implícita, pues a nadie se le escapa ya el papel que éstas juegan en el desarrollo de valores necesarios e imprescindibles para un desarrollo profesional que incluya una vertiente comprometida, crítica, reflexiva, activa y humana (Soler, 2008). Este trabajo se orienta a evidenciar, a través de la investigación educativa, lo que a menudo en la literatura en relación con el ApS se presenta como intuición o bien como construcción teórica.

\section{Aprendizaje Servicio y educación emocional}

El desarrollo emocional es imprescindible (Bisquerra, 2005) para un/a docente que participe en una construcción social y escolar más justa y equitativa. No son pocos quienes reclaman que la educación emocional, debe estar presente en nuestras aulas (Sala, Abarca y Marzo, 2002; Sánchez-Román y Sánchez-Calleja, 2015) y en la formación del profesorado (Bisquerra, 2010, Palomero, 2009). En nuestro sistema educativo no se fomentan momentos de toma de conciencia y en nuestra sociedad tampoco. El modelo educativo reproduce las exigencias y los apresurados tiempos de la posmodernidad, los momentos de reflexión (tan importante en el hecho educativo) son anulados por el número de exigencias a las que nos vemos sometidos. Prima la cantidad obviando o dando de lado la calidad, que tan a menudo tiene que ver con la articulación de la justicia social o la inclusión. El ApS nos devuelve educativamente voces, tiempos, reflexiones, compromiso y cuestionamiento del statu quo.

El descubrimiento de que existe interacción entre ApS y educación emocional tampoco es nuevo, como señalábamos con anterioridad. Podemos encontrar referencias en la literatura científica al hecho de que el ApS contribuye al desarrollo personal y social de las personas que participan de él. Los trabajos de Eberly y RocheOlivar (1979), Billig (2000), Conway, Amel y Gerwien (2009), Folgueiras, Luna y Puig (2013), García-Pérez y Mendía (2015) apuntan en este sentido, si bien no se centran de manera explícita en las relaciones entre ApS y la educación emocional.

Atendiendo a lo descrito por Puig, Batlle, Bosch y Palos (2009), el Aprendizaje y Servicio se configura como una propuesta educativa que:

(...) incide en el aprendizaje de contenidos intelectuales, afectivos y de comportamiento: desarrolla competencias aplicables a diversos ámbitos vitales; aviva el pensamiento crítico y la responsabilidad cívica, transmite valores y virtudes que fomentan el desarrollo personal y la ciudadanía, y contribuye a mejorar el entorno social, así como las instituciones implicadas en el proyecto. (p. 22)

Dicho de otra manera, articula un currículum que conecta cognición y emoción en los términos expresados por Goleman (1995) y los reconcilia (Folgeiras, Luna y Puig, 2013). Y es que el "aproximarse al Aprendizaje y Servicio supone un descubrimiento apasionado del estar, el hacer y el ser con sentido público en educación" (García y Cotrina, 2015, p. 9). Es de suma importancia que tomemos conciencia de lo que somos, de lo que hacemos y de cómo nos vamos construyendo, creciendo y desarrollando. 
Si el ApS desarrolla competencias emocionales, podemos afirmar que estamos educando emocionalmente, porque el fin de la educación emocional es precisamente este desarrollo competencial (Bisquerra, 2003). Si actualmente la educación emocional aparece como una temática de interés, no lo es menos en la formación del profesorado (Bisquerra, 2005; Palomero, 2009), si bien habría que especificar que la mayoría de las aproximaciones se realizan desde la denominada inteligencia emocional. Podríamos decir respecto a esta última, que es un término más reconocido a nivel mundial que el de educación emocional. Fue enunciado por primera vez en 1990 por los autores Salovey y Mayer, parece que al principio pasó desapercibido hasta que fue popularizado en 1995 por Goleman, a través de su best-seller. Como podemos ver, es un término bastante reciente sobre el que, como sucede en la mayoría de los casos, no existe unanimidad en cuanto a su definición, dada la diversidad de corrientes y perspectivas, centradas principalmente en considerarla como una habilidad o como un rasgo de personalidad (Pérez, Petrides y Furnham, 2005). En nuestro caso, lo entendemos como constructo capaz de ser modificado (Agulló, Filella, Soldevilla y Ribes, 2011; Goleman, 1996 y Güel, 2013), de ahí que aparezca la educación emocional. Nos decantamos por este término, que nos parece más didáctico y pedagógico y que está más en consonancia con lo que venimos preconizando en este trabajo.

Cuando hablamos del auge del ApS, descubrimos con frecuencia que, en gran medida se debe a los beneficios que aporta al ámbito educativo debido a su potencial para la construcción del aprendizaje en primera persona fomentando el compromiso, la ética y la responsabilidad social. Esto permite (Bisquerra, 2008; Puig, 2009) desarrollar una serie de competencias emocionales que hacen posible el aprender a ser (autoconocimiento, autoestima, conciencia emocional, autogestión, motivación, etc.) y el aprender a convivir (habilidades sociales, empatía, habilidades de vida y bienestar, etc.), dos de los cuatro pilares de la educación que recoge el Informe Delors (1996). En esta misma línea, desde la lectura de dos de dichos pilares, encontramos las aportaciones de Mendía (2012) que desarrolla la relación entre ApS y el ámbito personal o aprender a ser, lo cual ilustra apuntando que favorece "el pensamiento crítico, resolución de problemas, actitudes positivas hacía el aprendizaje, liderazgo, independencia" (p. 74). Asimismo hace notar la relación entre el Aps y el ámbito social o aprender a convivir, lo que, en palabras del autor supone "la apreciación de sistemas de valores opuestos, valoración de las diferencias culturales y habilidades de preocuparse y cuidar de otros" (Mendía, 2012, p. 74).

Este trabajo, a diferencia de otros, se centra en las relaciones entre ApS y educación emocional. Para ello se ha realizado una revisión de la literatura que ha permitido el avance de un marco conceptual a través del que poder empezar a pensar en el desarrollo de competencias emocionales a través de propuestas de Aprendizaje y Servicio en la formación inicial del profesorado. Dicho marco de trabajo supone un espacio construido a través de la integración de aportaciones de distintos autores, lo que le otorga singularidad. Asume los planteamientos de Bisquerra (2010) quien define las competencias emocionales como "conjunto de conocimientos, capacidades, habilidades y actitudes necesarias para tomar conciencia, comprender, expresar y regular de forma apropiada los fenómenos emocionales". (p. 19). Este autor identifica cinco competencias emocionales (Bisquerra, 2007, p. 70) "conciencia emocional, 
Tabla 1. Marco conceptual "teórico" de desarrollo de la educación emocional a través del Aprendizaje y Servicio

\begin{tabular}{|c|c|c|c|c|c|}
\hline DIMENSIÓN & COMPETENCIA & DESCRIPCIÓN & ASPECTOS & $\begin{array}{c}\text { ¿Se } \\
\text { desarrolla } \\
\text { en ApS? }\end{array}$ & $\begin{array}{c}\text { PILARES } \\
\text { DE LA } \\
\text { EDUCACIÓN }\end{array}$ \\
\hline \multirow{15}{*}{ Intrapersonal } & \multirow{4}{*}{$\begin{array}{l}\text { Conciencia } \\
\text { Emocional }\end{array}$} & \multirow{4}{*}{$\begin{array}{l}\text { Capacidad para tomar } \\
\text { conciencia de lo que } \\
\text { siente como persona y } \\
\text { de lo que sienten los/ } \\
\text { as demás, así como el } \\
\text { clima emocional de un } \\
\text { contexto determinado. }\end{array}$} & $\begin{array}{l}\text { Toma conciencia de las propias } \\
\text { emociones. }\end{array}$ & $x$ & \multirow{15}{*}{$\begin{array}{l}\text { Aprender } \\
\text { a ser }\end{array}$} \\
\hline & & & Da nombre a las emociones. & & \\
\hline & & & $\begin{array}{l}\text { Comprende las emociones de los } \\
\text { demás. }\end{array}$ & $x$ & \\
\hline & & & $\begin{array}{l}\text { Toma conciencia de la interacción } \\
\text { entre emoción, cognición y } \\
\text { comportamiento. }\end{array}$ & & \\
\hline & \multirow{4}{*}{$\begin{array}{l}\text { Regulación } \\
\text { Emocional }\end{array}$} & \multirow{4}{*}{$\begin{array}{l}\text { Capacidad para utilizar } \\
\text { las emociones de } \\
\text { forma adecuada para } \\
\text { responder al contexto o } \\
\text { situación. }\end{array}$} & Expresión emocional apropiada. & $X$ & \\
\hline & & & $\begin{array}{l}\text { Regulación de emociones y } \\
\text { sentimientos. }\end{array}$ & $X$ & \\
\hline & & & Habilidades de afrontamiento. & $x$ & \\
\hline & & & $\begin{array}{l}\text { Competencia para autogenerar } \\
\text { emociones positivas. }\end{array}$ & & \\
\hline & \multirow{7}{*}{$\begin{array}{l}\text { Autonomía } \\
\text { Emocional }\end{array}$} & \multirow{7}{*}{$\begin{array}{l}\text { Incluye un conjunto } \\
\text { de capacidades } \\
\text { relacionadas con la } \\
\text { autogestión emocional. }\end{array}$} & Autoestima. & $x$ & \\
\hline & & & Automotivación. & $x$ & \\
\hline & & & Autoeficiencia emocional. & $x$ & \\
\hline & & & Responsabilidad. & $x$ & \\
\hline & & & Presenta una actitud positiva. & & \\
\hline & & & $\begin{array}{l}\text { Es capaz de realizar un análisis } \\
\text { crítico de normas sociales. }\end{array}$ & $x$ & \\
\hline & & & Resiliencia. & & \\
\hline \multirow{15}{*}{ Interpersonal } & \multirow{9}{*}{$\begin{array}{l}\text { Competencia } \\
\text { Social }\end{array}$} & \multirow{9}{*}{$\begin{array}{l}\text { Capacidad para } \\
\text { mantener buenas } \\
\text { relaciones con otras } \\
\text { personas. }\end{array}$} & Domina las HH.SS. básicas. & $x$ & \multirow{9}{*}{$\begin{array}{l}\text { Aprender a } \\
\text { convivir }\end{array}$} \\
\hline & & & Respeta a los demás. & $x$ & \\
\hline & & & Practica la comunicación receptiva. & $X$ & \\
\hline & & & Practica la comunicación expresiva. & $x$ & \\
\hline & & & Comparte emociones. & $x$ & \\
\hline & & & $\begin{array}{l}\text { Tiene un comportamiento prosocial } \\
\text { y cooperación. }\end{array}$ & $x$ & \\
\hline & & & Asertividad. & $x$ & \\
\hline & & & Previene y soluciona conflictos. & $x$ & \\
\hline & & & $\begin{array}{l}\text { Tiene capacidad para gestionar } \\
\text { situaciones emocionales. }\end{array}$ & $x$ & \\
\hline & \multirow{6}{*}{$\begin{array}{l}\text { Habilidades de } \\
\text { vida y bienestar }\end{array}$} & \multirow{6}{*}{$\begin{array}{l}\text { Capacidad para adoptar } \\
\text { comportamientos } \\
\text { apropiados y } \\
\text { responsables para } \\
\text { afrontar las diferentes } \\
\text { situaciones que se } \\
\text { puedan dar en los } \\
\text { distintos contextos en } \\
\text { los que se desenvuelve. }\end{array}$} & Fija objetos adaptativos. & $x$ & \multirow{6}{*}{$\begin{array}{l}\text { Aprender a } \\
\text { hacer } \\
\text { Aprender a } \\
\text { aprender }\end{array}$} \\
\hline & & & Toma decisiones. & $X$ & \\
\hline & & & Busca ayuda y recursos. & $x$ & \\
\hline & & & $\begin{array}{l}\text { Ejerce una ciudadanía activa y } \\
\text { participativa, crítica y responsable y } \\
\text { comprometida. }\end{array}$ & $X$ & \\
\hline & & & Bienestar emocional. & & \\
\hline & & & Fluir. & $X$ & \\
\hline
\end{tabular}

Fuente: Elaboración propia, partiendo de las aportaciones de Bisquerra, (2007 y 2010), Carpena (2010) y Delors (1996). 
regulación emocional, autonomía emocional, competencia social y habilidades de vida y bienestar", que tomaremos como referentes. Por su parte, las aportaciones de Carpena (2010), nos permiten introducir un elemento categorial adicional de las competencias emocionales, las dimensiones intrapersonal e interpersonal (o social). La primera alude al conjunto de competencias emocionales cuyo desarrollo permite al sujeto tomar conciencia de sí mismo; y la segunda al conjunto de las que posibilitan "tomar conciencia de uno mismo en constante interacción con el entorno" (Carpena, 2010, p. 48). Además, hay que tener en cuenta que ambas dimensiones son complementarias y acontecen de manera interactiva. El marco conceptual se completa con otra categoría más, la que ofrecen los pilares de la educación (Delors, 1996) asociados a la educación emocional. Completa este marco los resultados del análisis de la literatura científica en relación con Aprendizaje y Servicio y éste en la formación inicial del profesorado. El resultado ha sido expresado en forma de tabla para una compresión más gráfica.

\section{Metodología}

Esta investigación se circunscribe al Grado de Educación infantil de la Universidad de Cádiz, y en concreto a la asignatura "Fundamentos Pedagógicos de las necesidades Educativas en la Infancia", que desarrolla bajo una lógica de Aprendizaje y Servicio, adscrita a un proyecto denominado "Institucionalización curricular del Aprendizaje-Servicio en los títulos de Educación".

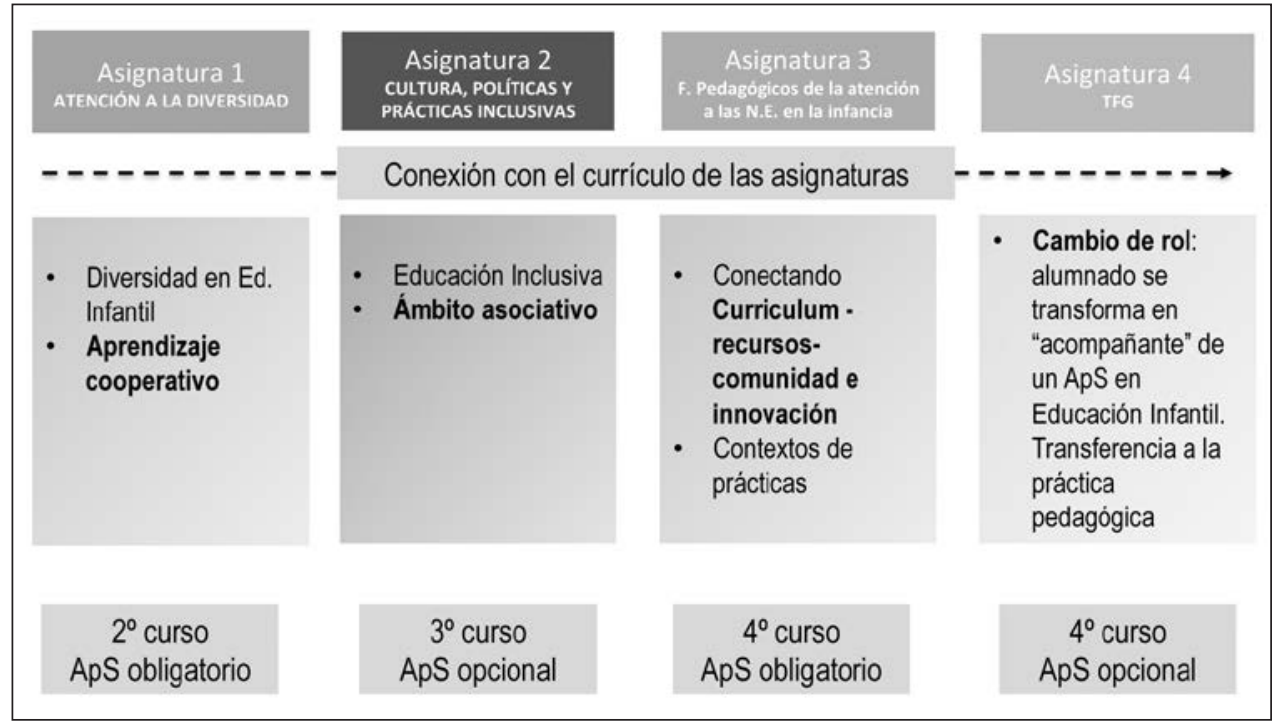

Figura 1. Itinerario curricular articulado a través de ApS en el Grado de Educación Infantil de la UCA

Dicho proyecto se ha orientado a comprender las aportaciones que puede proporcionar el ApS a la formación inicial de los/as futuros/as docentes de forma general y, 
en particular, en qué medida interfiere y contribuye en su formación inclusiva (García y Cotrina, 2012 y 2015). Para ello, se establece como eje metodológico el ApS en tres asignaturas del Grado de Educación Infantil, concretamente para los cursos de $2^{\circ}, 3^{\circ}$ y $4^{\circ}$, dentro de un itinerario curricular que se desarrolla de manera simultánea y progresiva para los tres cursos y concluye en una cuarta asignatura, el Trabajo Fin de Grado. En lo simultáneo, nos referimos a que en los tres cursos se desarrolla esta metodología, no siendo condición imprescindible para el alumnado de $4^{\circ}$ haberla utilizado y vivenciado en cursos anteriores. Lo hacemos así con la finalidad de no privarles en el conocimiento, desarrollo y evaluación de la misma. En lo progresivo, hacemos alusión a las competencias que ponemos en juego, dado el nivel de desarrollo, formación y maduración del alumnado en los diferentes cursos. De ahí que, en $2^{\circ}$ nos centremos en las competencias referidas a la organización como equipos cooperativos. En $3^{\circ}$, al tratarse de una optativa de la mención "Educación inclusiva", las competencias se encaminan a las claves culturales, políticas y prácticas de la educación inclusiva y al conocimiento profundo de alianzas en el entorno. En $4^{\circ}$, lo que buscamos es el desarrollo de competencias enfocadas al diseño y desarrollo de proyectos de corte inclusivo, lo que se complementa con un cambio de rol del estudiante cuando éste decide desarrollar un ApS inclusivo como Trabajo Fin de Grado.

Tabla 2. Resumen de experiencias de ApS desarrolladas durante el curso 2014-15 en el itinerario curricular

\begin{tabular}{lcccc}
\hline \multicolumn{1}{c}{ ASIGNATURA } & CURSO & $\begin{array}{c}\mathrm{N}^{\circ} \\
\text { EXPERIENCIAS } \\
\text { DE APS }\end{array}$ & $\begin{array}{c}\mathrm{N}^{\circ} \text { DE } \\
\text { ESTUDIANTES } \\
\text { IMPLICADOS-AS }\end{array}$ & $\begin{array}{c}\mathrm{N}^{\circ} \text { ENTIDADES } \\
\text { CORTENARIADO }\end{array}$ \\
\hline $\begin{array}{l}\text { Atención a la Diversidad } \\
\text { en Ed. Infantil }\end{array}$ & $2^{\circ}$ & 1 & 198 & 3 \\
\hline $\begin{array}{l}\text { Cultura, políticas y } \\
\text { prácticas inclusivas en Ed. } \\
\text { Infantil }\end{array}$ & $3^{\circ}$ & 9 & 45 & 29 \\
\hline $\begin{array}{l}\text { Fundamentos pedagógicos } \\
\text { de las necesidades }\end{array}$ & $4^{\circ} \mathrm{A}$ & 14 & 54 & 34 \\
\cline { 2 - 6 } \begin{tabular}{l} 
educativas en la infancia \\
\cline { 2 - 6 }
\end{tabular} & $4^{\circ} \mathrm{B}$ & 12 & 58 & 39 \\
\hline Trabajo Fin de Grado & $4^{\circ}$ & 13 & 55 & 35 \\
\hline
\end{tabular}

Los resultados de dicho proyecto, que ampliamente ilustran la consecución del objetivo del mismo, apuntaron nuevas líneas de indagación, entre las que destacan la articulación de la voz del alumnado a través del ApS y el desarrollo de las competencias emocionales en la formación inicial del profesorado a través del ApS. Este trabajo se adscribe a esta última línea que se desarrollada a través del proyecto "Haciendo visible el desarrollo de competencias emocionales a través del Aprendizaje y Servicio" y que se articula atendiendo al desarrollo metodológico expresado en la figura 2.

Este artículo presenta, a partir de distintos apartados, los resultados de las tres primeras fases, que han sentado las bases para la construcción de un instrumento evaluativo que permita valorar el desarrollo de competencias emocionales a través del Aprendizaje y Servicio. Si el marco conceptual de referencia, expresado en el apar- 
tado anterior, supone la concreción de la Fase 1, los siguientes apartados se referirán a la Fase 2, que, de manera específica supone el análisis de la voz del alumnado expresada a través de sus producciones que permiten hacer emerger un marco práctico.
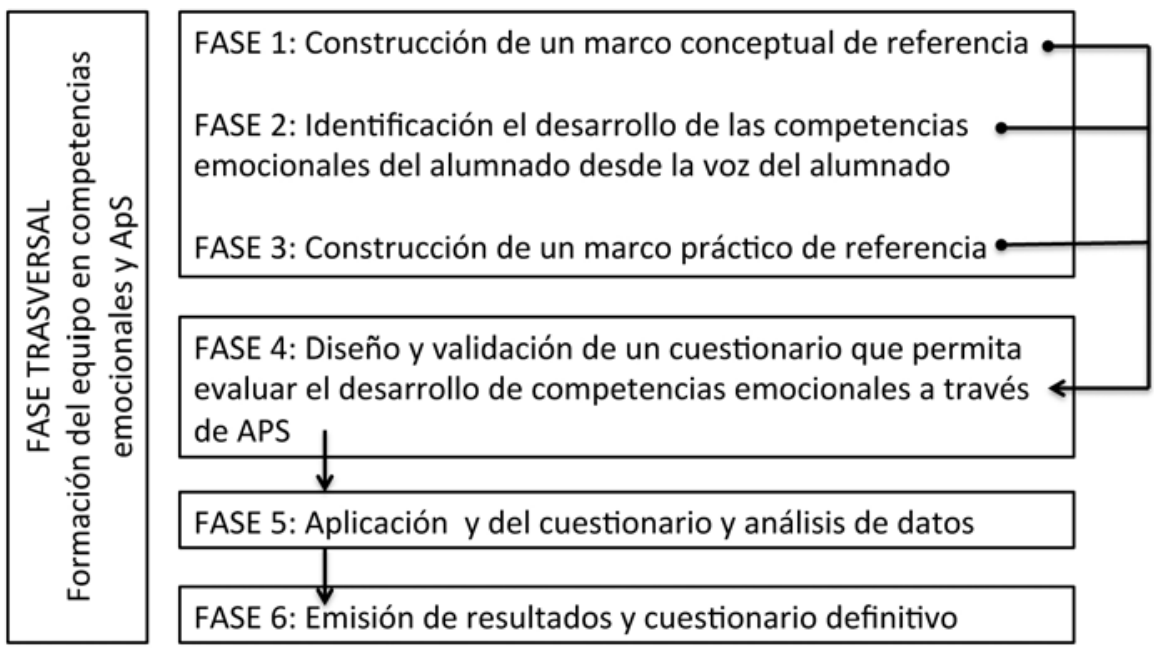

Figura 2. Desarrollo metodológico en fases del proyecto Haciendo visible el desarrollo de competencias emocionales a través del Aprendizaje y Servicio

Esta indagación se sitúa en una perspectiva interpretativa, de corte fenomenológico (Wolcott, 1992) en cuanto que se orienta a la descripción y comprensión de los significados vividos. En este sentido, hemos querido escuchar a nuestro alumnado desde la reflexión, quien después de participar en una experiencia de ApS enriquecida de procesos de autogestión, presenta de manera individual y colectiva informes experienciales. La reflexión en el ApS (Páez y Puig, 2015) constituye un ingrediente privilegiado en cuanto que "incrementa la fuerza educativa de cada uno de los dinamismos del Aprendizaje y Servicio y refuerza los vínculos entre dichos dinamismos (...) Da sentido personal y social a las actividades". (p. 15). De este modo, la importancia de este aspecto en el contexto de esta investigación viene dada por la coherencia entre el propio objeto de estudio (el Aprendizaje y Servicio) y su objetivo, donde son las propias voces las que permiten identificar el desarrollo a nivel personal y social, aspectos muchas veces relegados en nuestras aulas por dar prioridad al cognitivo. Los relatos personales permiten la incorporación de que somos un todo, una globalidad: cuerpo, mente, emoción y espíritu y que todo ello, está conectado e interrelacionado (Sánchez Calleja y Sánchez Román, 2016).

Este trabajo, de manera específica, se orienta a identificar y comprender si el alumnado participante en ApS, en $4^{\circ}$ curso del Grado en Educación Infantil de la Universidad de Cádiz, expresa y reconoce el desarrollo de competencias emocionales a través de su participación en dichas experiencias. En el conjunto de la investigación supone un primer paso para contrastar el marco conceptual que emerge de la revisión 
de la literatura y las investigaciones previas en relación con el ApS y la Educación Emocional; así como adquiere un carácter evaluativo en el contexto de nuestra propuesta didáctica que nos proporciona evidencias investigativas para andamiar tanto los procesos de reflexión del alumnado (a través del cuestionario que emergerá como resultado del proyecto completo), como el enriquecimiento didáctico de los diseños y los procesos que se ponen en acción a través de las propuestas específicas de ApS.

Este trabajo se centra en las experiencias vividas por alumnado de $4^{\circ}$ curso. Es decir, los/as informantes están concluyendo su formación en el Grado de Educación Infantil. Nos centramos en dos de los tres grupos donde se ha tenido acceso a la información generada de forma individual y grupal de manera completa (grupos A y C). Como queda presentado en la tabla 2, el alumnado participante se concreta en 109 personas que forman 27 grupos de trabajo. Las fuentes de información han sido documentales, de manera específica los informes individuales (109) y grupales (27) que elaboran al final de la asignatura. Todos ellos fueron analizados cualitativamente siguiendo el procedimiento de Miles y Huberman (1994), a través de un proceso de análisis de contenido. De este forma, los relatos de los/as estudiantes, como fuente de información, fueron: a) transformados a partir de los archivos originales entregados por el alumnado, b) reducidos a unidades de análisis, a través de su codificación en categorías, y c) listados y organizados para emitir resultados. El marco de referencia de dicho análisis fue el sistema categorial expresado en la tabla 1. Hay que señalar que, si bien se contemplaba inicialmente la posibilidad de construcción de categorías emergentes, ello no fue necesario al no identificarse mediante el análisis dicha situación (es decir, no hubo nuevas categorías). Considerados todos los informes, identificamos que solo 15 estudiantes, de manera individual, aludían entre sus aprendizajes, a alguna/s de las competencias emocionales expresadas en nuestro marco conceptual de referencia. Sin embargo, en el caso de los informes grupales lo hacían 19 grupos de los 27. Así pues, podemos adelantar que en las reflexiones de carácter grupal la dimensión emocional de los aprendizajes emerge de una manera más frecuente que cuando nuestro estudiante se expresa de manera individual.

\section{Resultados}

Como se ha expresado en el apartado anterior, el análisis de los relatos de nuestros/as estudiantes hacen pensar en un desarrollo emocional a través de la participación en experiencias de Aprendizaje y Servicio. Pero, ¿en qué sentido? En primer lugar, podemos hacer notar que tanto en el caso de los relatos individuales como en los grupales emergen referencias las dimensiones intrapersonales e interpersonales aunque las segundas de una manera más intensa, tanto para el caso de los informes personales como el de los grupales. Ello puede apreciarse en la tabla posterior.

También cabe destacar para cada uno de los análisis (individual y grupal) la identificación de la presencia de las cinco competencias emocionales, si bien en los relatos grupales se identifican más aspectos de cada una de ellas, así como con mayor intensidad y frecuencia. Pasamos a continuación a presentar e ilustrar de voz (ejemplos) los resultados diferenciando entre las expresiones en informes individuales y grupales. 
Tabla 3. Análisis de datos y comparativa con el marco conceptual de referencia de relación entre ApS y Educación Emocional

\begin{tabular}{|c|c|c|c|c|c|c|}
\hline & & & $\begin{array}{c}\text { Plano } \\
\text { conceptual }\end{array}$ & & $\begin{array}{l}\mathrm{z} \text { del } \\
\text { nnado } \\
\text { cipante }\end{array}$ & \\
\hline DIMENSIÓN & COMPETENCIA & ASPECTOS & $\begin{array}{c}\text { ¿Se } \\
\text { desarrolla } \\
\text { en ApS? }\end{array}$ & Indv & Grupal & \multirow{15}{*}{$\begin{array}{l}\text { Aprender } \\
\text { a ser }\end{array}$} \\
\hline \multirow{15}{*}{ Intrapersonal } & \multirow{4}{*}{$\begin{array}{l}\text { Conciencia } \\
\text { Emocional }\end{array}$} & $\begin{array}{l}\text { Toma conciencia de las propias } \\
\text { emociones. }\end{array}$ & $x$ & $x$ & $x$ & \\
\hline & & Da nombre a las emociones. & & & $x$ & \\
\hline & & Comprende las emociones de los demás. & $x$ & & $x$ & \\
\hline & & $\begin{array}{l}\text { Toma conciencia de la interacción entre } \\
\text { emoción, cognición y comportamiento. }\end{array}$ & & $x$ & & \\
\hline & \multirow{4}{*}{$\begin{array}{l}\text { Regulación } \\
\text { Emocional }\end{array}$} & Expresión emocional apropiada. & $x$ & & & \\
\hline & & Regulación de emociones y sentimientos. & $x$ & $x$ & $x$ & \\
\hline & & Habilidades de afrontamiento. & $x$ & & & \\
\hline & & $\begin{array}{l}\text { Competencia para autogenerar } \\
\text { emociones positivas. }\end{array}$ & & & & \\
\hline & \multirow{7}{*}{$\begin{array}{l}\text { Autonomía } \\
\text { Emocional }\end{array}$} & Autoestima. & $x$ & $x$ & $x$ & \\
\hline & & Automotivación. & $x$ & & & \\
\hline & & Autoeficiencia emocional. & $x$ & & & \\
\hline & & Responsabilidad. & $x$ & $x$ & $x$ & \\
\hline & & Presenta una actitud positiva. & & $x$ & $x$ & \\
\hline & & $\begin{array}{l}\text { Es capaz de realizar un análisis crítico de } \\
\text { normas sociales. }\end{array}$ & $x$ & & & \\
\hline & & Resiliencia. & & & & \multirow{10}{*}{$\begin{array}{l}\text { Aprender } \\
\text { a convivir }\end{array}$} \\
\hline \multirow{15}{*}{ Interpersonal } & \multirow{9}{*}{$\begin{array}{l}\text { Competencia } \\
\text { Social }\end{array}$} & Domina las HH.SS. básicas. & $x$ & & & \\
\hline & & Respeta a los demás. & $x$ & $x$ & $x$ & \\
\hline & & Practica la comunicación receptiva. & $x$ & & $x$ & \\
\hline & & Practica la comunicación expresiva. & $x$ & $x$ & $x$ & \\
\hline & & Comparte emociones. & $x$ & $x$ & & \\
\hline & & $\begin{array}{l}\text { Tiene un comportamiento prosocial y } \\
\text { cooperación. }\end{array}$ & $x$ & & & \\
\hline & & Asertividad. & $x$ & & & \\
\hline & & Previene y soluciona conflictos. & $x$ & $x$ & $x$ & \\
\hline & & $\begin{array}{l}\text { Tiene capacidad para gestionar } \\
\text { situaciones emocionales. }\end{array}$ & $x$ & & & \\
\hline & \multirow{6}{*}{$\begin{array}{l}\text { Habilidades de } \\
\text { vida y bienestar }\end{array}$} & Fija objetos adaptativos. & $x$ & & $x$ & \multirow{6}{*}{$\begin{array}{l}\text { Aprender } \\
\text { a hacer } \\
\text { Aprender } \\
\text { a } \\
\text { aprender }\end{array}$} \\
\hline & & Toma decisiones. & $x$ & $x$ & $x$ & \\
\hline & & Busca ayuda y recursos. & $x$ & $x$ & $x$ & \\
\hline & & $\begin{array}{l}\text { Ejerce una ciudadanía activa y } \\
\text { participativa, crítica y responsable y } \\
\text { comprometida. }\end{array}$ & $x$ & $x$ & $x$ & \\
\hline & & Bienestar emocional. & & & & \\
\hline & & Fluir. & $x$ & & & \\
\hline
\end{tabular}




\section{Competencias emocionales desarrolladas a través de experiencias de ApS desde la perspectiva individual}

Como se anunciaba con anterioridad se alude a las cinco competencias emocionales, como puede apreciarse en la figura 3.

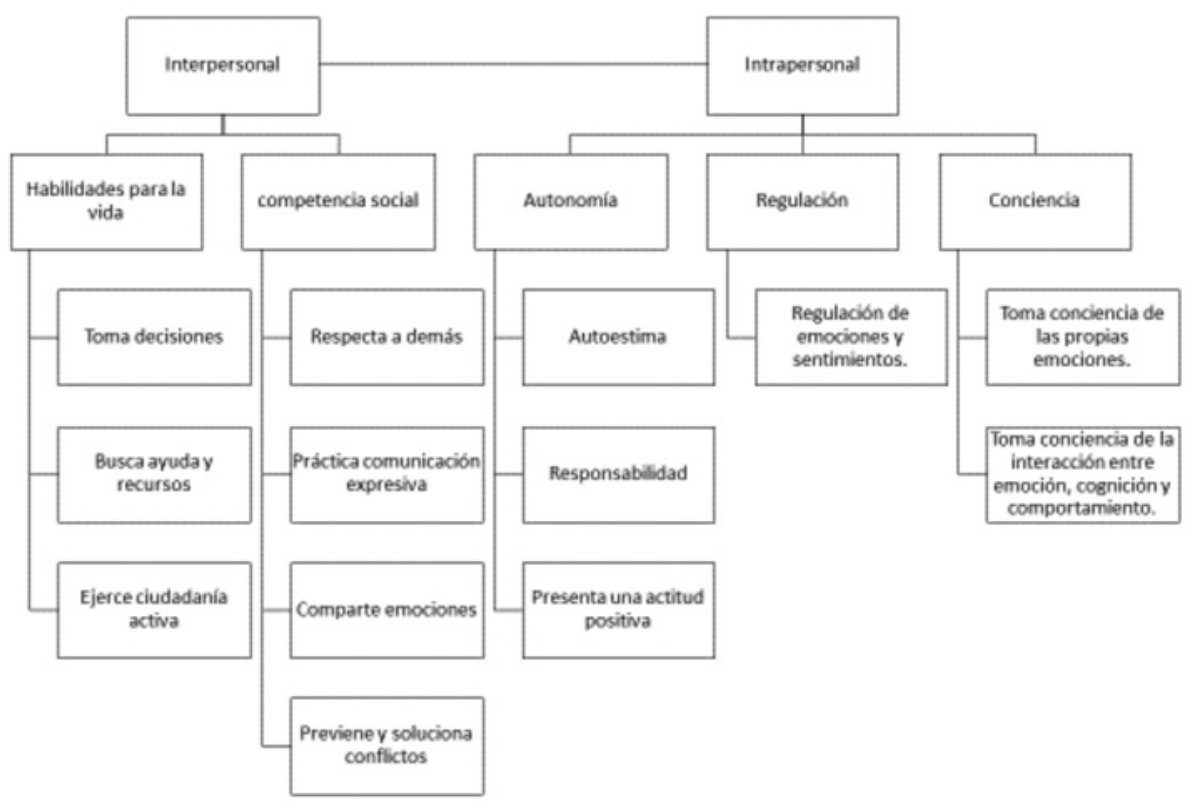

Figura 3. Competencias emocionales desarrolladas por los/as estudiantes participantes, a través de experiencias de ApS desde la perspectiva individual

En concreto, respecto al desarrollo de la conciencia emocional se hace referencia a cómo el ApS favorece la toma de conciencia:

"Tras esta experiencia me he dado cuenta que es muy importante conocerse a uno mismo para poder conocer a los demás. Es necesario expresar los sentimientos, puesto que es una manera más que tenemos las personas de comunicarnos". (A.3)

Así como ponen de manifiesto la importancia de la expresión emocional.

"Es fundamental saber expresar una emoción para recibir u ofrecer aquello que necesita en cualquier momento". (A.3)

En relación con la regulación emocional se apunta el valor de la gestión propia y de las interacciones grupales:

"Aprender a gestionar mis sentimientos y emociones ante distintas situaciones experimentadas en el centro y dentro de nuestro grupo". (A.4)

A través de las aportaciones, podemos resaltar tres aspectos que se identifican en el contexto de la autonomía emocional, competencia que aparece con mayor 
intensidad. En concreto lo referido a la autoestima, casi de la mayoría, a la responsabilidad y por último actitud positiva:

"Conocerme a mí misma ante determinadas situaciones, darme cuenta de mis limitaciones y mis posibilidades". (A.3)

"Desarrolla en la persona un sentimiento de pertenencia y unión al grupo, además contribuye a la formación del autoconcepto, autoimagen y una autoestima positiva". (A.8)

"Desarrollar el sentido de la responsabilidad". (A.15)

"Ser una persona más positiva valorando la posibilidad de hacer lo que hago y tener lo que tengo". (A.1)

La competencia social es también una de las que aparece con mayor frecuencia, identificándose en un sentido más amplio. Así, aspectos como el respecto a los demás y a sus ideas:

"A través de la tolerancia y con el ritmo de cada persona, aprendemos a ver las cosas desde otra perspectiva diferente. No por ello hay que juzgarlo, sino todo lo contrario, debemos respetarlo". (A.10)

La comunicación expresiva, la solución de conflictos y el compartir emociones. Son ilustrados ampliamente:

"Buscar soluciones a aquellos conflictos que se nos presentan, ya sea con nosotros mismos, con los compañeros, con el mismo servicio, con la alianzas..." (A.5)

"Comunicarme de manera clara para que los demás puedan entenderme". (A.3)

Se puede observar cómo desde el punto de vista del alumnado, en relación con la competencia habilidades de vida y bienestar, las experiencias de ApS vividas apuntan a la búsqueda de soluciones (como ayuda y recursos):

"Cuando por ejemplo, pierdes una alianza y el esquema planteado se te rompe, ¿dónde viene qué hacer? Esa es la parte que no existe en ningún papel. Ahí viene tu creatividad y tu participación, y tus ganas de sacar tu proyecto adelante". (A.5)

Además, implica un intenso papel la toma de decisiones y de manera importante una toma de conciencia de la propia profesión:

"Me ha servido para crecer como persona ya que el simple hecho de poder ayudar a tantas familias sin obtener nada material a cambio ha sido muy gratificante. También el Ilevarlo hasta la escuela, me ha hecho reflexionar y plantearme lo importante que es la figura del maestro para los futuros alumnos/as que tenga a mi cargo, el crear conciencia de los problemas existentes en la sociedad actual es tan importante como cualquier contenido didáctico". (A.6)

"He podido contrastar y observar el valor que tenemos las personas como recursos para poder ayudar en lo que se necesite. Asimismo, he conocido una metodología inclusiva, sobre la cual no tenía conocimiento y me ha enseñado a reconocer la importancia y lo imprescindible que son las alianzas y contactos, para un buen trabajo cooperativo". (A.10) 
Así como del valor del ejercicio de una ciudadanía activa, participativa, crítica y responsable y comprometida:

"Darme cuenta de que soy una persona solidaria, capacitada de habilidades para ayudar al otro, que no necesito que me instruyan una responsabilidad para implicarme en un proyecto, que soy útil para la sociedad, que soy capaz de ofrecer hasta lo que no tengo y para ver mi grado de empatía hacia el otro." (A.9)

\section{Competencias emocionales desarrolladas a través de experiencias de ApS desde la perspectiva grupal}

Como se señaló con anterioridad, cuando nuestros/as estudiantes contrastan de manera grupal sus reflexiones individuales se produce un análisis mucho más enriquecido en relación con las competencias emocionales que emergen del ejercicio de ApS. Ello puede apreciarse en la figura 4.

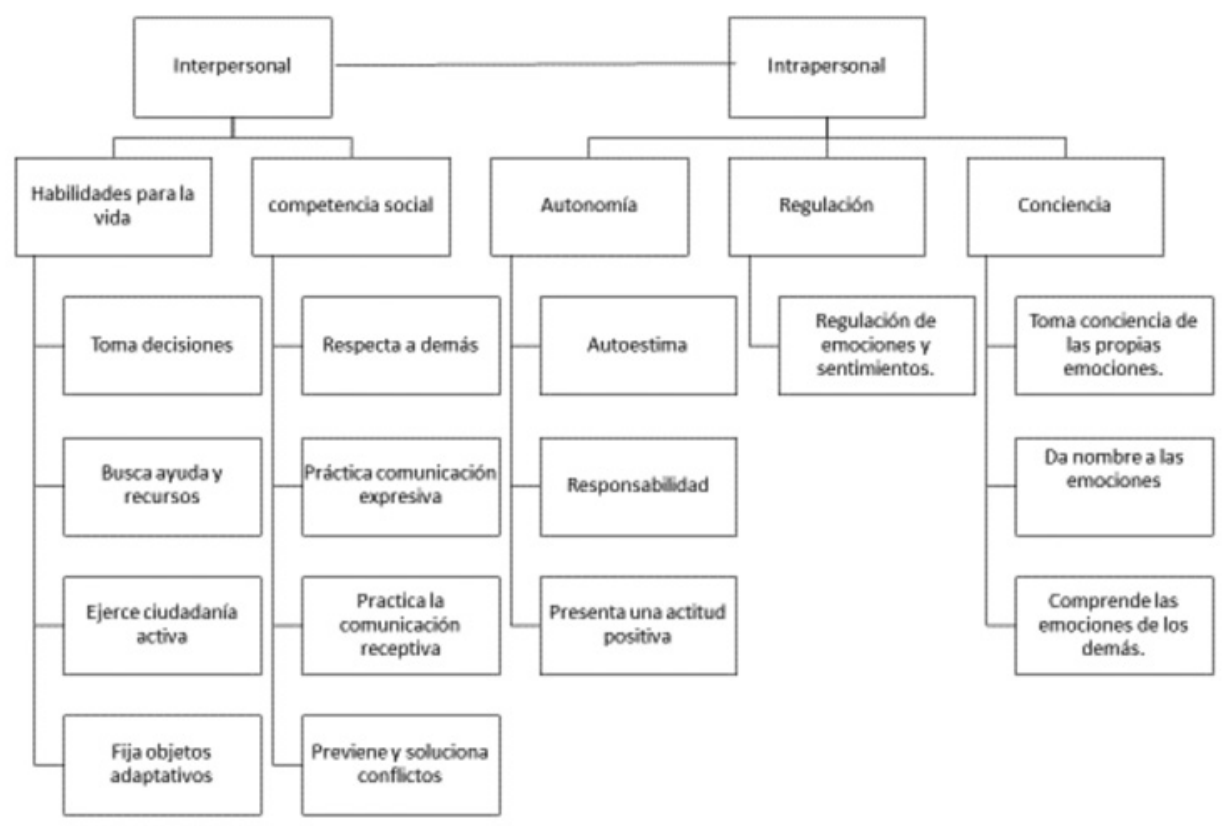

Figura 4. Competencias emocionales desarrolladas por los/as estudiantes participantes, a través de experiencias de ApS desde la perspectiva grupal

Respecto a la conciencia emocional, se pone de manifiesto importancia para ellos/as de nombrar las emociones:

"Ante situaciones de frustración, nuestras reacciones se focalizaron en analizar nuestras emociones a través de palabras, poniendo nombre a nuestros sentimientos e intentado reconducir hacia soluciones productivas". (G.3) 
Especialmente valoran la posibilidad de comprender a los/as demás y cómo toman una mayor conciencia de uno/a mismo/a:

"A comprender sentimientos ajenos". (G.4)

"Conocimientos de nosotros mismos, ampliando nuestro autoconcepto". (G.18)

El ApS en el contexto de la asignatura supone un ejercicio grupal y cooperativo y en este sentido se articulan procesos de regulación emocional (lo que aparece en las respuestas grupales: G.4, G.10, G.16, G.17 y G.18), identificando conductas primarias (ira), en su mayoría, aunque también aparecen las secundarias (vergüenza).

"Controlar la frustración y la rabia". (G. 12)

"A ser menos tímidas". (G.13)

Por otra parte, el alumnado declara que el ApS ha contribuido en el desarrollo de su autonomía emocional. Identifican aspectos como el aumento de la autoestima, la capacidad de análisis crítico, y la emergencia de la responsabilidad:

"Ha habido un cambio en la percepción de nosotras mismas y de nuestras capacidades". (G.8)

"Nos hemos convertido probablemente en personas con una visión algo más crítica de las necesidades sociales". (G.5)

"Crecer como personas responsables". (G.14)

La competencia social articulada a través de la convivencia resulta ser una competencia altamente reconocida en su experiencia por el alumnado:

"Se convive y aprendes a respetar a los demás, a escuchar diferentes ideas, opiniones y a solventar problemas buscando diferentes alternativas". (G.6)

Destacan en este sentido, la resolución de conflictos, la aceptación de las diferencias, la comunicación expresiva y receptiva y, en su mayoría, la oportunidad de compartir emociones:

"Compresión y tolerancia hacia los demás". (G.8)

"A escuchar a todas las compañeras y a comunicarnos eficazmente". (G.4)

"Importancia de una buena comunicación". (G.18)

“Diálogo y expresión de cómo nos sentimos, Ilegando a conectar nuestras emociones para poder ayudarnos". (G.3)

Finalmente, hay que indicar que ilustran ampliamente el desarrollo de habilidades de vida y bienestar que implican la toma de decisiones enriquecida, el fijar objetivos adaptativos colectivos y la búsqueda de soluciones (ayuda y recursos):

"La toma de decisiones conjunta produce resultados de mayor calidad y nos enriquecemos de las ideas de las otras". (G.1) 
"Establecer objetivos colectivos por encima de los individuales". (G.1)

"Buscar soluciones y superar cada barrera dada". (G.5 y G.14)

Pero, especialmente destacan el desarrollo de habilidades para ejercicio de una ciudadanía activa y participativa, crítica, responsable y comprometida:

"Hemos vivenciado sentimientos de ayuda, unión, colaboración, solidaridad, compromisos, etc.". (G.11)

\section{DisCUSIÓN Y CONCLUSIONES}

Son numerosas las voces que destacan la importancia del desarrollo emocional en los/as docentes. Así mismo, existe consenso en apuntar el vínculo entre el Aprendizaje y Servicio y desarrollo emocional (Eberly y Roche-Olivar, 1979; Billig, 2000; Conway, Amel y Gerwien, 2009), Folgueiras, Luna y Puig, 2013 o García-Pérez y Mendía, 2015), como apuntábamos al inicio de este artículo. Este trabajo ha ilustrado de manera específica que es posible una orientación del Aprendizaje y Servicio, como metodología inserta en la formación inicial del profesorado, que contribuya a una educación emocional docente.

Los resultados de esta aproximación investigativa, ilustrada por la voz del alumnado, apuntan que, a través de la propuesta pedagógica articulada en la asignatura, nuestro alumnado trabaja y desarrolla esos aspectos emocionales que, por considerarse transversales y que son responsabilidad de todos/as, ninguno/a le damos espacio-tiempo en nuestras aulas. Además desarrolla valores que humanizan y que consideramos necesarios e imprescindibles para el desarrollo social, profesional y personal, reconciliando cognición y emoción (Folgeiras, Luna y Puig, 2013). Nos referimos a responsabilidad individual y social, respeto, tolerancia, solidaridad, generosidad, entre otros. En el aspecto social, se fomenta la cohesión y se abandona y rechaza la competitividad, fomentando la colaboración y cooperación, compartiendo e implicándonos. En referencia a lo individual, se fomenta la creatividad y el pensamiento divergente en cuanto a las búsqueda de alternativas para atender a la necesidad detectadas, así como el empoderamiento, el alumnado ocupa su lugar, se siente útil y capaz de hacer y de transformar, cree en él, en sus posibilidades y recursos personales, se siente bien con lo que hace, aumentando así su autoestima.

Mendía (2012) apunta que el Aprendizaje y Servicio,

Incluye en el desarrollo de los aprendizajes la gestión de las emociones, la motivación, el conocimiento de la otra persona (sus sentimientos, su situación personal...). Dimensiones relacionadas con la autoestima y el estado de ánimo positivo, que procuran el bienestar emocional personal y colectivo. (p. 76).

Aspectos, que en su mayoría, hemos visto reflejados a través de la voz de nuestro alumnado. Evidenciamos con esta investigación que también es posible en el contexto de la formación inicial del profesorado. Además, la práctica del ApS en esta propuesta atiende a la diversidad en el sentido en que todas las propuestas son válidas y cada persona lo hace desde lo que es, lo que ha experimentado en su desarrollo y desde su propia construcción del conocimiento, lo que supone el desarrollo de una 
educación más inclusiva (García y Cotrina, 2015). Es decir, de todo lo que lleva en esa mochila que ha ido preparando y equipando a lo largo de su desarrollo para enfrentarse a los diversos retos que le ofrece el día a día.

Esta primera aproximación a la comprensión de la emergencia de competencias emocionales a través del ApS en la formación inicial del profesorado se inserta en una propuesta de itinerario formativo, lo que nos ofrece posibilidades importantes, porque abre la vía de dicha comprensión más allá de una asignatura concreta. En este sentido sienta las bases de futuros trabajos que se orienten a la evaluación del programa y sus efectos a través de un estudio longitudinal.

Por otro lado, a pesar de lo limitado del estudio en este momento a dos grupos en un solo curso nos proporciona herramientas para la mejora del propio itinerario. En este sentido evidencia la necesidad de rediseñar los procesos de reflexión del alumnado (elemento fundamental en el ApS), puesto que se hace patente cómo los momentos de intercambio colectivo resultan más potentes, desde el punto de vista pedagógico y pueden volver a enriquecer la construcción personal. Así, si en este momento, el reflexivo sigue la secuencia individual-grupal, vemos la necesidad de dotar a éste de un nuevo ciclo individual que se asiente en el previo colectivo.

Además, nos informa de que tenemos el reto de enriquecer los procesos y contextos de ApS para que permitan emerger aspectos que el estudio arroja como no reconocidos por el alumnado, como la expresión emocional adecuada, las habilidades de afrontamiento, la automotivación, la autoeficiencia emocional, el dominio de habilidades sociales básicas, la prosocialidad, cooperación y asertividad y el análisis crítico de las normas sociales, todos ellos aspectos emocionales ampliamente reconocibles en propuestas de ApS.

Queremos concluir posicionándonos en el convencimiento de lo interesante que resulta el Aprendizaje y Servicio como metodología en la formación inicial del profesorado, algo que ya enunciaban en sus trabajos Campo (2006), Francisco y Moliner (2010) o García y Cotrina, (2016). Apuntando además que, en particular, nos permite el trabajo y desarrollo de las competencias emocionales; contribuyendo, por tanto, al desarrollo integral de futuros/as maestros/as para que ellos/as puedan, a su vez, favorecer y fomentar este desarrollo en alumnado, sufragando la construcción de un mundo más humano y más inclusivo. En el aquí y en el ahora, estas propuestas les permiten vivir una experiencia real en primera persona, mirando la escuela desde la participación activa siendo realmente protagonistas y no meros espectadores/as. (Santos Guerra, 2000).

\section{REFERENCIAS BIBLIOGRÁFICAS}

Agulló, M. J., Filella, G., Soldevila, A. y Ribes, R. (2011). Evaluación de la educación emocional en el ciclo medio de Educación Primaria. Revista de Educación, 354, 765-783.

Batlle, R. (2011). ¿De qué hablamos cuando hablamos de aprendizaje-servicio? Crítica, 972, 49-54.

Batlle, R. (2013). El aprendizaje-servicio en España: el contagio de una revolución pedagógica necesaria. Madrid: PPC. 
Billig, S. (2000). Service-Learning Impacts on Youth, Schools and Communities: Research on K-12 School-Based Service-Learning, 1990-1999. Denver, CO: RMC Research Corporation.

Bisquerra, R. (2003). Educación emocional y competencias básicas para la vida. Revista de investigación educativa, 21(1), 7-43.

Bisquerra, R. (2005). La educación emocional en la formación del profesorado. Revista Interuniversitaria de Formación del Profesorado, 19, 95-114.

Bisquerra, R. (2008). Educación para la ciudadanía y convivencia. El enfoque de la educación emocional. Madrid: Wolters Kluwer.

Bisquerra, R. (coord.) (2010). La educación emocional en la práctica. Barcelona: Cuadernos de Educación.

Bisquerra, R. y Pérez- Escoda, N. (2007). Las competencias emocionales. Educación $X X 1,10,61-82$.

Campo, L. (2010). El aprendizaje servicio en la universidad como propuesta pedagógica. En Martínez (ed.), Aprendizaje Servicio y responsabilidad social de las universidades. (pp. 81-92). Madrid: Octaedro.

Carbonell Sebarroja, J. (2015). Pedagogías del siglo XXI. Alternativas para la innovación educativa. Barcelona: Graó.

Conway, J., Amel, E. y Gerwien, D. (2009). Teaching and learning in the social context: A meta-analysis of service learning's effects on academic, personal, social, and citizenship outcomes. Teaching of Psychology, 36, 233-245.

Delors, J. (1996). Los cuatro Pilares de la educación. En La Educación Encierra un tesoro. Informe a la UNESCO de la Comisión internacional sobre la educación para el Siglo XXI. (pp. 91-103). Madrid: Santillana/UNESCO.

Eberly, D. J. y Roche-Olivar, R. (1979). Aprendizaje-servicio y prosocialidad. Ethology and Sociobiology, 1, 3-11.

Folgueiras, P. y Martínez, M. (2009). El desarrollo de competencias en la universidad a través del Aprendizaje y Servicio Solidario. Revista Interamericana de Educación para la Democracia (RIED), 2(1), 55-76.

Folgueiras, P., Luna, E. y Puig-Latorre, G. (2013). Aprendizaje y servicio: estudio del grado de satisfacción de estudiantes universitarios. Revista de Educación, 362, 159-185.

Fredericks, L. (2003). Making the Case for Social and Emotional Learning and ServiceLearning. Paper 1. Recuperado de: http://digitalcommons.unomaha.edu/slceslgen/1.

Francisco, A. y Moliner, L. (2010). El Aprendizaje Servicio en la Universidad: una estrategia en la formación de la ciudadanía crítica. Revista Electrónica Interuniversitaria de Formación del Profesorado, 13(4), 69-77.

Furco, A. (2001). Advancing Service-Learning at Research Universities. New Directions for Higher Education, 114, 67-78.

García, M. y Cotrina, M. (2012). La contribución de la universidad al desarrollo de prácticas educativas inclusivas: dilemas y propuestas para avanzar compartiendo. Revista de Educación Inclusiva, 5(1), 123-138.

García, M. y Cotrina, M. (2015). El Aprendizaje y Servicio en la formación inicial del profesorado: de las prácticas educativas críticas a la institucionalización curricular. Profesorado, Revista de curriculum y formación del profesorado, 19(1), 8-25. 
García, M. y Cotrina, M. (2016). Aprendizaje y servicio: una herramienta pedagógica inclusiva para $<<$ vivir $>>$ la transformación en el marco de la formación inicial del profesorado. En Fernández Navas, M. y Alcaraz, N. (coord.), Innovación educativa: más allá de la ficción. (pp. 175-187). Madrid: Pirámide.

Greenberg, L. (2000). Emociones: una guía interna. Bilbao: Desclée De Brouwer.

Goleman, D. (1996). Inteligencia emocional. Barcelona: Kairós.

Güel, M. (2013). ¿Tengo inteligencia emocional? Barcelona: Paidós.

Mangas, S. L. y Martínez-Odría, A. (2012). La implantación y difusión del Aprendizaje-Servicio en el contexto educativo español. Retos de futuro de una metodología de enseñanza-aprendizaje para promover la innovación en la Educación Superior. Revista del Congrés Internacional de Docència Universitària i Innovació (CIDUI), 1(1).

Martínez, M. (ed.) (2010). Aprendizaje servicio y responsabilidad social de las universidades. Barcelona: Octaedro.

Martínez-Domínguez, B., Domínguez, I. M., Sáez, I. A. y Amundarain, M. G. (2015). El aprendizaje-servicio, una oportunidad para avanzar en la innovación educativa dentro de la Universidad del País Vasco. Tendencias Pedagógicas, 21, 99-118.

Miles, M. B. y Huberman, A. M. (1994). Qualitative data analysis. A new sourcebook of methods. (pp. 429-444). Beverly Hills: Sage.

Mendía, R. (2012). Aprendizaje-Servicio como una estrategia inclusiva para superar las barreras al aprendizaje y a la participación. Revista Educación Inclusiva, 5(1), 71-82.

Murillo, J. M. y Aramburuzabala, P. (2014). Aprendizaje-servicio y justicia social. Cuadernos de pedagogía, 450, 50-53.

Opazo, H., Araburuzabala, P. y Cerrillo, R. (2016). A review of the situation of service-learning in higher education in Spain. Asia-Pacific Journal of Cooperative Education, 17(1), 75-91.

Palomero, P. (2009). Desarrollo de la competencia social y emocional del profesorado: una aproximación desde la psicología humanista. Revista Electrónica Interuniversitaria de Formación del Profesorado, 12(2), 145-153.

Páez, M. y Puig, J. M. (2015). La reflexión en el Aprendizaje-Servicio. Revista internacional de educación para la justicia social, 2(2), 13-32.

Palos, J. y Puig, J. M. (2006). Rasgos pedagógicos del aprendizaje-servicio. Cuadernos de pedagogía, 357, 60-63.

Pérez, J. P., Petrides, K. V. y Furnham, A. (2005). Measuring Trait Emotinal Intelligence. En R. Schulze y R. D. Roberts (eds.), Emotional Intelligence: An Internacional Handbook. Ashland, OH: Hogrefe \& Huber.

Pérez Escoda, N., Filella, G. y Soldevilla, A. (s.f.). Competencias emocionales y habilidades sociales en estudiantes universitarios. Revista electrónica de Motivación y Emoción, XIII, 34. Recuperado de: http://reme.uji.es/articulos/numero34/article1/ texto.html.

Puig Rovira, J. M. (2009). Aprendizaje servicio (ApS): educación y compromiso cívico. Barcelona: Graó. 
Puig Rovira, J. M. (2007). Aprendizaje servicio: educar para la ciudadanía. Madrid; Barcelona: Octaedro.

Rubio, L., Prats, E. y Gómez, L. (coord.) (2013). Universidad y sociedad. Experiencias de Aprendizaje servicio en la universidad. Barcelona: Universitat de Barcelona (Institut de Ciències de I'Educación). Recuperado de: http://angelsull.es/sostenibilidad/ wp-content/uploads/2013/04/TEXTO-PROYECTOS-ApS-UNIVERSIDAD.pdf.

Rubio, L. (coord.) (2008). Guía zerbikas 0: aprendizaje y servicio solidario, guía de bolsillo. Bilbao: Fundación Zerbikas.

Sala, J., Abarca, M. y Marzo, L. (2002). La educación emocional y la interacción profesor/a-alumno/a. Revista electrónica interuniversitaria de formación del profesorado, 5(3), 1-4.

Sánchez, L., García, M. y Benítez, R. (2015). Evaluación del Aprendizaje-Servicio en el itinerario curricular de Educación Infantil de la Universidad de Cádiz. VI Congreso Nacional y I Internacional ApS (u)6. Universidad de Granada, 29 y 30 de mayo de 2015 (en prensa).

Sánchez Calleja, L. y Sánchez Román, A. (2016). La educación emocional: un cambio de mirada. Una propuesta desde lo emergente y vivencial. En Fernández Navas, M. y Alcaraz, N. (coords.), Innovación educativa: más allá de la ficción. (pp.159172). Madrid: Pirámide.

Sánchez Román, A. y Sánchez Calleja, L. (2015). Educación emocional: diez razones para una propuesta. Cuadernos de Pedagogía, 452, 86-91.

Santos Guerra, M. A. (2000). La participación es un árbol. Revista Kikiriki. Cooperación Educativa, 55-56, 105-116.

Soler Villalobos, M. (2008). De la educación socioemocional a la educación en valores. Madrid: Ministerio de Educación y Ciencia.

Tapia, M. N. (2014). La aportación del aprendizaje-servicio en el mundo. ¿De qué calidad educativa hablamos? Cuadernos de Pedagogía, 450, 54-56.

Tedesco, J. C., Hernaiz, I., Tapia, M. N. y Rial, S. (2008). El aprendizaje-servicio en la educación superior: Una mirada analítica desde los protagonistas. Buenos Aires: Editorial Universitaria de Buenos Aires.

Wolcott, H. (1992). Posturing in qualitative research. En LeCompte, W. y Preissle, J. (eds.), The handbook of qualitative research in education (pp. 3-44). San Diego, CA: Academic Press. 